\title{
Blutige Grenzüberschreitungen
}

Vampirismus, Liminalität und Migration

Sanglants franchissements de frontières : vampirisme, liminalité et migration Bloody Border Crossings: Vampirism, Liminality and Migration

\section{Clemens Ruthner}

\section{OpenEdition}

\section{Journals}

\section{Édition électronique}

URL : https://journals.openedition.org/ceg/10187

DOI : 10.4000/ceg. 10187

ISSN : 2605-8359

\section{Éditeur}

Presses Universitaires de Provence

\section{Édition imprimée}

Date de publication : 20 janvier 2020

Pagination : 213-220

ISBN : 979-10-320-0253-7

ISSN : 0751-4239

Référence électronique

Clemens Ruthner, „Blutige Grenzüberschreitungen“, Cahiers d'Études Germaniques [Online], 78 | 2020,

Online erschienen am: 28 Oktober 2021, abgerufen am 01 Februar 2022. URL: http://

journals.openedition.org/ceg/10187 ; DOI: https://doi.org/10.4000/ceg.10187 


\title{
Blutige Grenzüberschreitungen \\ Vampirismus, Liminalität und Migration
}

\author{
Clemens RUTHNER \\ Trinity College Dublin
}

Hélène Barrière gewidmet

Die Mundhöhle ist ein Ort finsterer Geheimnisse. ${ }^{1}$ Sie ist nicht nur gleichsam ein Futteral für das Gebiss, sondern auch Vorhölle für das Essen: ein liminaler Zwischenraum zwischen dem Körperinneren und der Umwelt, also jenem nichtidentischen Draußen und der abtötenden Verschlingung und Verdauung des Fremden drinnen, seinem Gegessen-Werden, aus dem Eigenes wird bzw. das Eigene am Leben gehalten wird.

Das Blecken des Gebisses gehört aber auch zu den Urszenen des Vampirfilms, für den die Fangzähne des untoten Blutsaugers ja erst konzipiert wurden ${ }^{2}$, spielt doch das Dentale bei den Vampiren der Literatur weniger eine Rolle - wohl aber das Blut, eingedenk des alten Glaubens der Antike, wonach der rote Saft Sitz und Treibstoff des Lebens ist, der selbst Tote anzutreiben vermag (wenn wir an das 11. Buch der Odyssee denken, wo Kirke mit Hilfe von geschlachteten Schafen die Toten beschwört). Das Göttliche des Blutes bedingt auch das alte Verbot in zwei von drei abrahamitischen Religionen, es zu verzehren. Die dritte schließlich begeht die Transgression, wonach in der Kommunion das Blut des Gottessohnes getrunken und in einer kannibalischen Restfunktion sein Fleisch verzehrt wird - ob symbolisch oder real in der Transsubstantiation, bleibt den jeweiligen Theologen überlassen. Dieser verdrängte dionysische Exzess des Abendmahls geschieht, damit eine besondere Gemeinschaft entstehe, einerseits zwischen den Essenden untereinander, aber auch andererseits mit dem Aufgefressenen selbst. Man muss hier unwillkürlich an die idiomatische Phrase denken, wonach man „jemanden zum Fressen gern“ hat - ist doch das Verspeisen eine der intensivsten körperlichen Vereinigungen mit einem Objekt der Begierde, die man sich vorstellen kann, ja die ultimative Grenzüberschreitung.

Auch wenn es nun gewisse strukturelle Ähnlichkeiten zwischen dem Phantasma des Kannibalen und des Vampirs gibt, so frisst letzterer aber bekanntlich kein

1. Vgl. Hartmut Böhme, Beate Slominski (Hrsg.), Das Orale. Die Mundhöhle in Kulturgeschichte und Zahnmedizin, München, Fink, 2013.

2. Vgl. Stefan Keppler, Michael Will (Hrsg), Der Vampirfilm. Klassiker des Genres in Einzelinterpretationen, Würzburg, Könighausen \& Neumann, 2006. 
Fleisch, sondern es ekelt ihm davor; wohl aber trinkt oder saugt er Blut als eigentümliches Negativbild zu unserem Heiland, der ja streng genommen ebenso ein Untoter ist. Diese Transgression gehört zur Aufrechterhaltung jenes Schwebezustands des Vampirs zwischen Leben und Tod, für dessen Heuristik das kulturtheoretische Konzept des ,Liminalen“ vorgeschlagen werden soll, das sich vom britischen Anthropologen Victor Turner herleitet. ${ }^{3}$

Liminalität bezeichnet eine Zwischenposition in jener Transition, die Turner von Arnold van Genneps Rites de passage 4 übernommen hat: Übergangs- bzw. Initiationsriten etwa zwischen Kindheit und Erwachsensein, wie sie man in vormodernen Gesellschaften vielfach finden kann, in einer Schwundstufe aber auch noch in unserer Matura, der Reifeprüfung, die das Verlassen der Schule ins Leben formalisiert. ,Liminal' ist dann jene Schwelle zwischen zwei abgegrenzten Zuständen, ein zeitweiliges Dazwischen in einem Nicht-Mehr und Noch-Nicht. In dieser Phase werden die Betroffenen meist von normaler Sozietät separiert, ausgegrenzt, bevor ihnen rituell der Wiedereintritt in die bzw. eine neue Gemeinschaft gewährt wird. Turner schreibt:

\begin{abstract}
Van Gennep hat gezeigt, daß alle Übergangsriten drei Phasen aufweisen: die Trennungs-, die Schwellen- und die Angliederungsphase. In der ersten Phase (der Trennung) verweist symbolisches Verhalten auf die Loslösung eines Einzelnen oder einer Gruppe von einem früheren fixierten Punkt der Sozialstruktur, von einer Reihe kultureller Bedingungen (einem „Zustand“) oder von beidem gleichzeitig. In der mittleren „Schwellenphase“ ist das rituelle Subjekt (der „Passierende“) von Ambiguität gekennzeichnet; es durchschreitet einen kulturellen Bereich, der wenig oder keine Merkmale des vergangenen oder künftigen Zustands aufweist. In der dritten Phase (der Angliederung und Wiedereingliederung) ist der Übergang vollzogen. Das rituelle Subjekt - ob Individuum oder Kollektiv - befindet sich wieder in einem relativ stabilen Zustand und hat demzufolge anderen gegenüber klar definierte, sozialstrukturbedingte Rechte und Pflichten. ${ }^{5}$
\end{abstract}

Der Vampir indes - so lautet eine zentrale These dieses Beitrags - ist in der Zwischenwelt einer „Schwelle“ gefangen; ein beliebtes Filmmotiv zeigt immer wieder, dass er diese auch faktisch nicht überschreiten kann (und hier sei an eine These Hans Richard Brittnachers erinnert, der kompatibel mit Turner und van Gennep in der phantastischen Literatur oft die Handlungsstruktur einer „gescheiterten Initiation“ verwirklicht sieht ${ }^{6}$ ). Liminal ist jener Blutsauger, der

3. Vgl. Victor Turner, Das Ritual. Struktur und Anti-Struktur, übers. u. mit einem Nachwort v. M. Schomburg-Scherf, Frankfurt a. M./ New York, Campus, 2005 [1969]. Zur kritischen Konzeptualisierung in den Kultur- und Sozialwissenschaften vgl. Manuel Aguirre, Roberta Quance, Philip Sutton (Hrsg.), Margins and Thresholds. An Enquiry into the Concept of Liminality, Madrid, The Gateway Press, 2000; Jochen Achilles, Roland Borgards, Brigitte Burrichter (Hrsg.), Liminale Anthropologie. Zwischenzeiten, Schwellenphänomene, Zwischenräume in Literatur und Philosophie, Würzburg, Königshausen \&t Neumann, 2012; Agnes Horvath, Bjorn Thomassen, Harald Wydra (Hrsg.), Breaking Boundaries. Varieties of Liminality, New York/ Oxford, Berghahn, 2015.

4. Arnold van Gennep, Les Rites de passage. Étude systématique des rites, Paris, Picard, 1909 (dt. Übers.: Übergangsriten, aus dem Franz. von Klaus Schomburg und Sylvia M. SchomburgScherff, Frankfurt a. M., Campus, 2005).

5. Turner, Das Ritual, S. $94 f$.

6. Hans Richard Brittnacher, „Gescheiterte Initiationen. Anthropologische Dimensionen der literarischen Phantastik“, in Clemens Ruthner, Ursula Reber, Markus May (Hrsg.), Nach 
aus der südosteuropäischen Folklore in die Literatur und den Film migriert ist, dabei gleich in mehrfacher Hinsicht. ${ }^{7}$

Zunächst einmal ist er weder lebendig noch tot, sondern, wie der Privatgelehrte Montague Summers in den 1920er Jahren schreibt, „living in death“, wofür sich der paradoxe Begriff „untot“ eingebürgert hat:

A demon has no body, although for purposes of his own he may energize, assume, or seem to assume one, but it is not his real and proper body. So the vampire is not strictly a demon, although his foul lust and horrid propensities be truly demonic and of hell. Neither may the vampire be called a ghost or phantom, strictly speaking, for an apparition is intangible. The vampire has a body and his craving for blood is to obtain sustenance for that body. He is neither dead nor alive; but living in death. He is an abnormality; the androgyne of the phantom world; a pariah among the fiends. How fearful a destiny is that of the vampire who has no rest in the grave but whose doom it is to come forth and prey upon the living. ${ }^{8}$

So sehr aus der Defınition von Summers der katholische Möchtegern-Geistliche mit seinen eigenen Hemmschwellen spricht ${ }^{9}$, so fasst sie doch erstmalig die Liminalität des Vampirs in Worte. Denn das phantasmatische Wesen aus Folklore und Unterhaltungskultur stellt auch die abendländische Leitdichotomie von Leib und Seele in Frage, ist es doch kein Gespenst, sondern ein wandelnder Leichnam, in dessen ,Leben“ - d.h. vor allem: in seiner verstörenden Körperlichkeit - sich andere Konzepte der Beseelung als der christliche Dualismus ausdrücken mögen. ${ }^{10}$

Drittens vermengt der Vampir auch die kulturell meist klar voneinander abgegrenzten Bereiche von Ernährung und Fortpflanzung: Er bedarf keiner genital induzierten Zeugung und Geburt zu seiner Vermehrung, sondern eines Prinzips der Ansteckung, denn er gibt meist mit seinem Biss seine untote Teleologie weiter an sein Opfer.

Das bedeutet letztlich auch (viertens), dass er in Sachen Geschlecht eigentlich liminal - oder trans - sein müsste, ist dieses doch lediglich Ornament, aber keine biologische Notwendigkeit für ihn/sie (es?). Trotzdem ist es bemerkenswert, wie das untote Geschlecht erst jüngst - mit dem schwedischen Film Let the Right One In (Regie: Tomas Alfredson, 2008) - nachhaltig in Frage gestellt wurde, betonen doch sonst die meisten Vampirtexte und -filme eher stur das Gender des Vampirs etwa als dämonischer Aristokrat oder femme fatale. Ein Hauch dieser

Todorov. Neuere Zugänge zu einer Definition des Phantastischen in der Literatur, Tübingen, Francke, 2006, S. 15-30. Zur Phantastik als Literatur des Liminalen vgl. Clemens Ruthner, „Zur Theorie der Liminalität, oder: Die Grenzwertigkeit der Fantastik“, in Mario Grizelj (Hrsg.), Der Schauer(roman): Formen - Diskurszusammenhänge - Funktionen, Würzburg, Könighausen \& Neumann, 2010 (Film - Medien - Diskurs, 27), S. 77-90.

7. Vgl. Clemens Ruthner, „Vampir“, in Hans Richard Brittnacher, Markus May (Hrsg.), Phantastik. Ein interdisziplinäres Handbuch, Stuttgart, Metzler, 2013, S. 493-500.

8. Montague Summers, The Vampire. Its Kith and Kin, New York, Dorset, 1991 [1928], S. 1.

9. Vgl. Frederick S. Frank, Montague Summers. A Bibliographical Portrait, London, The Scarecrow Press, 1988.

10. Wie dies ein französischer Forscher für den ,historischen“ ,Vampir‘ (Wiedergänger, Nachzehrer) der Frühneuzeit behauptet; vgl. Claude Lecouteux, Geschichte der Gespenster und Wiedergänger im Mittelalter, Köln/ Wien, Böhlau, 1987; ders.: Die Geschichte der Vampire. Metamorphose eines Mythos, übers. von Harald Ehrhardt, Düsseldorf/ Zürich, Artemis \& Winkler, 2001. 
geschlechtlichen Liminalität findet sich freilich auch in Bram Stokers DraculaRoman, wo in einer denkwürdigen Szene der Vampir Mina Harker von seiner Brust zu trinken gibt. ${ }^{11}$

Last, but not least fließt aber auch die Bedeutung des Vampirs wie das Blut seiner Opfer, das eine Kontaktzone zwischen beiden schafft - und den Blutsauger als floating signifier ausweist, der sich der Fixierung mit semantischen Grenzpfählen zu widersetzen scheint, wie Brittnacher in seiner Ästhetik des Horrors schreibt:

Da Gewalttätigkeit und Blutdurst im Vampirmotiv buchstäblich aus dem Nichts kommen, dient sich seine literarische Adaption grundsätzlich jeder beliebigen Kritik an: Der Vampir erscheint mal als Sinnbild einer entmachteten und rachsüchtigen Aristokratie, mal als Symbol nymphomanischer Weiblichkeit, mal als das eines maßlosen DonJuanismus, mal wird mit ihm der Stalinismus gebrandmarkt, mal das Franco-Regime, mal die Jesuiten, dann wieder sind es Bürokratie, venerische Krankheiten oder die Furcht vor neueren wissenschaftlichen Entdeckungen wie Hypnose und Magnetismus, die im Vampir ihr Bild gefunden haben. Eben diese Elastizität verbietet eine [...] simple Deutung. ${ }^{12}$

Auch diese elastische Leere des Motivs, die Vieldeutigkeit förmlich anzieht, gehört zu dem, was hier als die mehrfache Liminalität des Vampirs bezeichnet worden ist. Man könnte sie freilich auch beschreiben als Figur des ausgeschlossenen Dritten, als ein virtuelles tertium datur, oder man sieht den Vampir überhaupt als Dekonstruktivisten avant la lettre, der an den Leitdifferenzen der westlichen Kultur nagt. Der Vampir ist aber nicht nur durch die Transgression seiner Jagd und Tötung menschlicher Opfer ein Grenzverletzer. Er ist auch - und dieser Kalauer sei riskiert - ein geborener Migrant aus den Peripherien Europas, und dies schon von seinen allerersten Anfängen an. ${ }^{13}$

Die wichtigste Episode dieser Wanderung geht auf jene Zeit im ersten Drittel des 18. Jahrhunderts zurück, als der Westbalkan nach den Türkenkriegen zeitweilig von habsburgischen Truppen besetzt war. Diese sahen sich in den beiden serbischen Dörfern Medvedja und Kisiljevo mit merkwürdigen Phänomenen konfrontiert: Nach einer Reihe von unerklärlichen, aber sich häufenden Todesfällen fühlten sich nämlich die Bewohner und Bewohnerinnen von ihren Toten verfolgt, sie verwüsteten die Friedhöfe und wollten auswandern. Deshalb wird in beiden Fällen eine Untersuchungsmission österreichischer (Militär-)Mediziner vor Ort geschickt; ihre Berichte haben sich bis heute erhalten, es sind sozusagen die

11. Vgl. Bram Stoker, Dracula, hrsg. von Nina Auerbach und David J. Skal, New York/ London, Norton, 1997 [1897], Kap. XXI.

12. Hans Richard Brittnacher, Ästhetik des Horrors. Gespenster, Vampire, Monster, Teufel und künstliche Menschen in der phantastischen Literatur, Frankfurt a. M., Suhrkamp, 1994 (st 2397), S. 125; vgl. Susanne Pütz, Vampire und ihre Opfer. Der Blutsauger als literarische Figur, Bielefeld, Aisthesis, 1992, S. 148.

13. Vgl. dazu etwa Thomas Bohn, Der Vampir. Ein europäischer Mythos, Köln/ Weimar, Böhlau, 2016; Massimo Introvigne, La stirpe di Dracula. Indagine sul vampirismo dall'antichità ai nostri giorni, Mailand, A. Mondadori, 1997. 
$X$-Files der Aufklärung. ${ }^{14}$ Mit diesen migrierte der Begriff dessen, was ein Vampir ist, aus der Wildnis des Balkans in die Zentren des frühaufgeklärten Europa: Wien, Halle, Leipzig, Paris, London. Es ist gleichsam die Emigration, ja Invasion eines Phantasmas, das in der Literatur der europäischen Romantik äußerst wirkungsträchtig werden sollte. ${ }^{15}$

Pikant daran ist, dass jener Kulturtransfer wahrscheinlich auf ein interkulturelles Rauschen in den Kommunikationskanälen zurückgeht: Dürften doch die österreichischen Militärärzte in ihrer Unkenntnis der serbischen Sprache wie auch der örtlichen Folklore das Wesen des Ungeheuers und seine Funktionsweise gründlich missverstanden haben, ebenso wie seinen möglicherweise korrumpierten Namen (den es vor 1732 in keinem Wörterbuch gibt) oder die Vorstellung, dass dieser ,Vampir Blut trinke. Diese Ernährungsweise dient ja unterschwellig auch dazu, die barbarische Herkunft des Vampirs aus bildungsfernen Gebieten xenophob zu stigmatisieren, indem er aus der Sicht der aufgeklärten Mittel- und Westeuropäer in die Nähe eines Menschenfressers rückt: ein Skandalon im zunehmend hygienisch re-organisierten Europa der Moderne, ebenso wie 250 Jahre später in der AIDS-Hysterie der Postmoderne, der Francis Ford Coppola mit seiner Verfilmung von Bram Stokers Dracula 1992 ein Denkmal gesetzt hat.

Dass Migration dem liminalen Vampir nicht akzidentiell zukommt, sondern zu seinen zentralen Bestimmungen zählt, wird klar, wenn man sich die weitere Kulturgeschichte des Phantasmas ansieht: Vampire und Vampirinnen werden immer wieder latent oder explizit mit jener Aura des Südost- oder Osteuropäischen versehen, die bedrohliche und latent gewalttätige Zivilisationsferne konnotiert. ${ }^{16}$ Nicht umsonst gab der amerikanische Komparatist Tomislav Longinović seiner imagologischen Untersuchung zu Serbien-Bildern in westlichen Diskursen den Horrorfilm-Titel Vampire Nation. ${ }^{17}$ Ebenso hält sich immer wieder das hartnäckige Gerücht, dass der amerikanische Kampfhubschrauber Bell AH-1 Cobra in einer lokalen Version unter dem Namen Dracula für die rumänische Luftwaffe produziert werden soll[te], als fliegender, aggressiver Angreifer with a local touch. ${ }^{18}$

14. Vgl. Bohn, ibid., sowie Clemens Ruthner, „Untotes Wachsen im Textgrab: Zur narrativen Ausarbeitung von Flückingers Vampirismus-Protokoll (1732) bei Herbert Mayo (1846)“, in Christoph Augustynowitsch, Ursula Reber (Hrsg.), Vampirglaube und magia postuma im Diskurs der Haburgermonarchie, Münster, LIT, 2011.

15. Vgl. Clemens Ruthner, „Untote Verzahnungen. Prolegomena zu einer Literaturgeschichte des Vampirismus“, in Julia Bertschik, Christa Agnes Tuczay (Hrsg.), Poetische Wiedergänger. Deutschsprachige Vampirismus-Diskurse vom Mittelalter bis zur Gegenwart, Tübingen, Francke, 2005, S. 11-42.

16. Vgl. Clemens Ruthner, „Süd/Osteuropäer als Vampire. Die Karriere Draculas vom blutrünstigen Tyrannen zum mythischen Blutsauger (Prolegomena zu einer Literaturgeschichte des Vampirismus II)“, in Kakanien revisited, www.kakanien.ac.at/beitr/fallstudie/CRuthner3.pdf [2003, Stand: 3. August 2018].

17. Vgl. Tomislav Z. Longinović, Vampire Nation. Violence as Cultural Imaginary, Durham, Duke Univ. Press, 2011.

18. Vgl. https://www.romania-insider.com/bell-helicopter-romania-may-produce-bell-attackhelicopters/ [2017, Stand: 3. August 2018]. 
Der Vampir dient aber schon seit dem Klassiker von Bram Stoker dazu, einer vagen Bedrohung aus dem ,Osten“ sein Gesicht zu leihen; dazu trägt das Dracula inhärente Invasions- und Seuchenphantasma bei, dem nur ein Inselbewohner des britischen Weltreiches so überzeugend Konturen verleihen konnte. Denn mehr als 100 Jahre vor Brexit ist Dracula ein alarmistischer Aufruf, ein geopolitischer call to arms am Vorabend des Ersten Weltkriegs, der die untote Migration aus Siebenbürgen (Transsylvanien) mit Vernichtung kontern möchte. Dieses Moment des ansteckenden Einfalls, das im Übrigen bei den Zombies noch viel ausgeprägter ist, gehört seither strukturbildend zum Vampir, der sich damit auch zur radikalen politischen Metapher eignet. ${ }^{19}$ Zum Sinnbild der Migration im heutigen Europa wurde der kleine Vampir im Übrigen auch im besagten schwedischen Film Let the Right One In, obwohl das dort explizit mit keinem Wort gesagt wird.

Migranten und ihre spezielle Liminalität spielen aber auch in der Realität des vampirischen Kulturbetriebs eine wichtige Rolle. Legendär geworden ist der Fall des ungarischen Schauspielers Bela Lugosi, der als Migrant nach New York kam, um dort Dracula zuerst am Broadway und später in Tod Brownings Kinofilm von 1931 seinen unvergleichlichen Akzent zu leihen. ${ }^{20}$ Angeblich konnte Lugosi anfänglich so wenig Englisch, dass man ihm seine Textzeilen in ungarischer Transkription hinschreiben musste und er oft gar nicht wusste, was er da sagte. Dieses latent orientalistische Prinzip wurde vor einigen Jahren vom Warschauer Teatr Rozmaitości (TR) quasi postkolonial umgedreht, als in der Nosferatu-Produktion von Grzegorz Jarzyna (2011) der Titelheld von einem deutschen Darsteller gespielt wurde, der kaum Polnisch konnte; auch sonst wurden Blick- und Bissrichtungen verkehrt, wahrscheinlich auch als Referenzen an Buffy, the Vampire Slayer - jene bahnbrechende Fernsehserie (WB Television Network, 1997-2003), wo sich die Blondine nicht mehr widerspruchslos zum Dummchen und vampirischen Nahrungsmittel degradieren lässt, sondern als Kampfsportlerin mit martial arts zurückschlägt.

Der erste Text freilich, der den Vampir - und speziell: Dracula - auf seinen Migrationsstatus hin befragte, war, wie es scheint, die kurze Erzählung Der Bettler vor dem Café Hippodrom; ebenso spielt die alternative und letztlich verschwindende Körperlichkeit des Blutsaugers hier eine zentrale Rolle, ist er doch gleichsam auch eine visible minority. Veröffentlicht wurde der Text $1970^{21}$, in der beginnenden Spätphase des Kalten Kriegs, von einem Südtiroler PhantastikAutor, der im Brotberuf Richter in München war: Herbert Rosendorfer (1934-2012).

19. Vgl. Clemens Ruthner, „Vampirism as Political Theory. From Voltaire to Alfred Rosenberg and Elfriede Jelinek“, in Alienne Becker (Hrsg.), Visions of the Fantastic. Proceedings from the 15th Int. Conference on the Fantastic in the Arts, Westport (CT)/ London, Greenwood Press, 1996 (Contributions to the Study of SF and Fantasy, 68), S. 3-11.

20. Vgl. Elisabeth Bronfen, „Das Kino als Vampir. Tod Brownings Dracula (1931)“, in Keppler, Will, Der Vampirfilm, S. 55-72.

21. Im Folgenden wird nach der Erstausgabe zitiert, in Herbert Rosendorfer, Der stillgelegte Mensch. Erzählungen, Zürich, Diogenes, 1970, S. 223-237. 
Sein Vampir ist Graf Dracula selbst, als arbeitsloser rumänischer Zuwanderer allerdings arg herabgekommen von einstiger Größe. Er fristet ein Dasein als Bettler vor dem im Titel genannten Café, in dem auch der Ich-Erzähler verkehrt und uns von Anfang an augenzwinkernd vermittelt, dass er wisse, um wen es sich hier handelt. Dies geschieht mithilfe von kleinen Andeutungen, die sich an die vampirkundige Lesegemeinde richten, wenn etwa von der bleichen Gesichtsfarbe des Mannes die Rede ist ${ }^{22}$, von seinem „Tobsuchtsanfall“23, als er eine Brücke überqueren muss (die oben angesprochene Schwellensituation), seiner Abneigung gegen die angeblich im Lokal servierte Knoblauchsuppe ${ }^{24}$, seinem biblischen Alter und ungarischen Akzent ${ }^{25}$.

Die Rahmenerzählung präsentiert eine Kriminalhandlung: der Bettler ist beim Versuch, in die Blutbank des örtlichen Krankenhauses einzubrechen, von der Polizei verhaftet worden. Am nächsten Tag, als die „klare Wintersonne“26 über dem Zellenfenster aufgegangen ist, ist er verschwunden; man findet nur noch „einen übelriechenden, schwarz-fettigen Belag auf dem Boden“27. Man muss nicht viel einschlägige Filme gesehen haben, um zu wissen, dass es sich hier um die klassischen Vampir-Rückstände handelt, die ein fast schon stereotypes abject ${ }^{28}$ des Horrorgenres darstellen.

Ebenso macht der Text die ethnische Stereotypierung des Vampirs mit - affirmativ oder ironisch, das sei einmal dahingestellt -, wenn es heißt:

\begin{abstract}
Wenn ein Osteuropäer erzählt, so ist das, als ob sich Steinmassen bewegten. Langsam, langsam beginnt es zu rollen, weit, weit greift es aus, kreist und greift um sich, immer weiter rollt es und bewegt sich, dreht sich in sich, bis es alles, alles erfaßt und das Weltrund, ja den Himmel in die alles umspannende, alles überschäumende Erzählung, unaufhaltsam gigantisch mahlende Erzählung- und Lügenfındlinge auftürmende, in das allgewichtige Geschehene der erzählten Geschichte einbezieht. ${ }^{29}$
\end{abstract}

Wie anhand dieses Textbeispiels deutlich wird, kommt Rosendorfers postmodern spielerischer Recycling-Fantastik - denn so kann man sie wohl am besten charakterisieren - nun auch eine gewisse satirische Qualität $\mathrm{zu}^{30}$, die in diesem Fall wohl zwei strategische Stoßrichtungen nimmt.

Zum einen haben wir es wohl mit einer Satire auf den Kalten Krieg zu tun, aus dem die Flüchtlinge kamen, die es in Deutschland und Österreich um 1970 spärlich, aber doch gab. Rosendorfers Dracula beschwert sich z.B., von allen historischen Vampirjägern seien die rumänischen Kommunisten wohl die

22. Rosendorfer, Bettler, S. 224 u. 228.

23. Ibid., S. 225.

24. Ibid., S. 231.

25. Ibid., S. 230.

26. Ibid., S. 226.

27. Ibid.

28. Vgl. dazu Julia Kristeva, Powers of Horror. An Essay on Abjection, New York, Columbia University Press, 1982.

29. Rosendorfer, Bettler, S. 231.

30. Vgl. das anderwärtig über H.C. Artmann Formulierte: Clemens Ruthner, „Dämon des Geschlechts. VampirInnen in der österreichischen Literatur nach 1945 (Bachmann, Artmann, Jelinek, Neuwirth u.a.)“, Modern Austrian Literature 31, 1998, H. 3-4, S. 65-88. 
schlimmsten gewesen ${ }^{31}$ - was historisch im Übrigen nicht richtig ist, ließ doch Ceaucescu im Zuge seines nationalkommunistischen Projekts dem historischen Territorialfürsten Vlad Dracula III. (1431-1476/77), auf den Stokers Roman u. U. lose Bezug nahm, eine eigene Briefmarke entwerfen und ein Denkmal errichten.

Wesentlich ist aber vor allem das Credo, dass sich nicht nur das Vampirgenre mit seinen diversen Versatzstücken abgenützt hat, sondern dass „,...] in einer Welt der Computer, der Girokonten und Supermärkte [...] kein Platz mehr für Vampire [ist]." ${ }^{32}$ Nicht zu vergessen, dass der alternde Dracula niemanden mehr tötet, sondern sich mit Blutwurst und Blutkonserven mehr schlecht als recht durchs Leben schlägt. Rosendorfers Erzählung wird damit zum Epitaph der in serieller Kommerzialisierung (wie etwa bei den britischen Hammer Films) verloren gegangenen Erhabenheit des Vampirs und seines Todes in der Spätmoderne.

Falsche Visionen entwickelt die kleine und vielleicht sogar ein klein wenig alberne Geschichte erst in unserem Zeitkontext: Hat sich doch der Ich-Erzähler ganz gründlich geirrt, dass sich Vampire in der Moderne und Postmoderne nicht gut halten. Ganz im Gegenteil, konnte doch Rosendorfer vielleicht in seinen letzten Lebensjahren noch den Siegeszug Stephenie Meyers und ihrer Twilight Saga durch die Beststellerlisten der Welt erleben. Auch die Eltern von shining Edward, jener Schwundstufe des einstmals sexuell aggressiven Vampirs, der jetzt absurderweise zum Kronzeugen des Sich-,Aufsparens' für die Hochzeitsnacht gemacht wird, sind übrigens nach Washington State zugezogen, also Migranten. Sein Körper ist freilich nicht mehr abweichend auffällig, sondern einfach nur schön; er zerfällt nicht zu stinkendem Matsch, sondern weiß sich zu beherrschen, und auch sein Grenzgängertum tritt nur in Extremsituationen zu Tage.

Der Migranten werden es in unseren Jahren allerdings immer mehr, sie konsumieren aber kein Blut mehr, sondern werden anderwärtig ausgegrenzt und dämonisiert. Ihre körperliche Präsenz wird zu der eines homo sacer ${ }^{33}$, und man muss sogar an Kafkas Verwandlung denken, wenn sie in Österreich (für 1,50 Euro pro Stunde) zum Borkenkäfersammeln herangezogen werden sollen. In der phantastischen Bilderwelt entsprechen dieser Angst vor den verelendeten Massen, seien es nun ein zorniges Proletariat, die medial verdummten Unterschichten der USA oder eben die Migrationsströme unseres Planeten, eher die Zombies, und diese ernähren sich anders: Sie schlagen ihre toten Zähne in das zuckende Fleisch ihrer noch lebenden Opfer und sie haben es vor allem auf ihre Gehirne abgesehen.

31. Rosendorfer, Bettler, S. 234.

32. Ibid., S. 235.

33. Vgl. Giorgio Agamben, Homo sacer. Die souveräne Macht und das nackte Leben, Frankfurt a. M., Suhrkamp, 1995. 